\title{
Design of a Microstrip Bowtie Antenna for Indoor Radio- Communications
}

\author{
Hector Fraga-Rosales, Mario Reyes-Ayala*, Genaro Hernandez-Valdez, Edgar Alejandro Andrade-Gonzalez, \\ Jose Raul Miranda-Tello, Felipe Alejandro Cruz-Perez and Sandra Lirio Castellanos-Lopez
}

Metropolitan Autonomous University, Electronics Department, 02200 Azcapotzalco, Mexico City, Mexico

\begin{abstract}
In this paper, a microstrip bowtie patch antenna (MBPA) for wireless indoor communications is carried out. Here, a microstrip transmission-line feed network was designed in order to match the MBPA. The proposed antenna uses a ground plane with the aim of narrowing down the back lobes in comparison with bowtie sheet antennas, which radiation pattern is omni-directional. The far-field pattern of the antenna was simulated using a finite-element numerical algorithm and obtained by interpolation employing nearfield equipment. The experimental results are described in detail intending to agree well with the simulated predictions. The antenna was designed, measured and built and its far field performance was evaluated with a $2.11 \mathrm{GHz}$ resonant frequency. The azimuth and elevation antenna patterns, antenna gain and, the matching frequency were the main parameters obtained to analyze the antenna behaviour. The antenna has a gain approximately equal to $8.77 \mathrm{dBi}$ and its beam-widths are higher than $100^{\circ}$ in $\mathrm{E}$ plane.
\end{abstract}

\section{Introduction}

Microstrip antennas have some advantages in order to be used in a lot of mobile communications systems [1-3]. The main advantages of this kind of antennas can be summarized as follows: they are compatible with mobile terminal equipment where a thin profile and a light weight might be necessary; they are relatively low-cost than other manufacturing techniques; their bandwidths are narrow; they have normally moderate quality factors; and, they have enough antenna gain, that is needed in the indoor radio-communications links [4-6].

Microstrip antennas can be made to emulate wire antennas; monopoles, dipoles, Yagi-Uda and logarithmic array antennas are widely configuration examples. Besides, rectangular and circular patches give a very narrow-band response approximately equal to $2 \%$. Then, bowtie antenna offers a moderate bandwidth (5\%) in comparison with rectangular and circular choices. Other approaches like fractal, slotted and defected structures have been developed intending to increase the bandwidth, but there are some tradeoffs to take into account, because this sort of antennas require a very high manufacturing accuracy, their electrical features are not stable and, its price is frequently more expensive [1-3, 7 9].

Bowtie antenna can be divided into two categories, sheet and microstrip patch antennas. Bowtie sheet antennas have omnidirectional radiation patterns and as a consequence of this, they have a lower antenna gain [1]. Thu use of ground planes in microstrip bowtie antennas changes the omnidirectional antenna pattern into a directional one.
In this paper, a Microstrip Bowtie Patch Antenna (MBPA) is presented and it is proposed for indoor communications systems. According to the considerations mentioned before, the MBPA is chosen in order to achieve a low-cost microstrip antenna with a 5\% bandwidth and a moderate antenna gain in a wide beamwidth. The antenna will be used in educational and low-cost mobile handsets or other terminal equipment for transportation vehicles; but, it will be almost used in low power radio applications.

The article is divided into four sections: the section one is an introduction to microstrip bowtie antennas; the section two is dedicated to describe the design methodology of the MBPA; in the third section of the paper, the main simulated and measured results are presented and discussed; and, the conclusions of this work are described in the section four.

\section{Design of the antenna}

The MBPA design is based on the triangular patch antenna theory. Uni-planar bowtie antennas like MBPA usually combine the use of two triangular antennas, but it is possible to use circular magnetic walls. This antenna can be built in a single side or in both sides of the Printed Circuit Board (PCB). In this work, a MBPA is designed and probed, this antenna uses a ground plane with the aim of achieving a directional radiation pattern with a very low back lobes, see the Figure 1 .

Each triangle has a non-perfect magnetic wall, where the effect of this wall can be estimated using empirical methods. The design procedure of the MBPA presented

Mario Reyes-Ayala:mra@ correo.azc.uam.mx 
here is based on the assumption that the triangles have perfect magnetic walls.

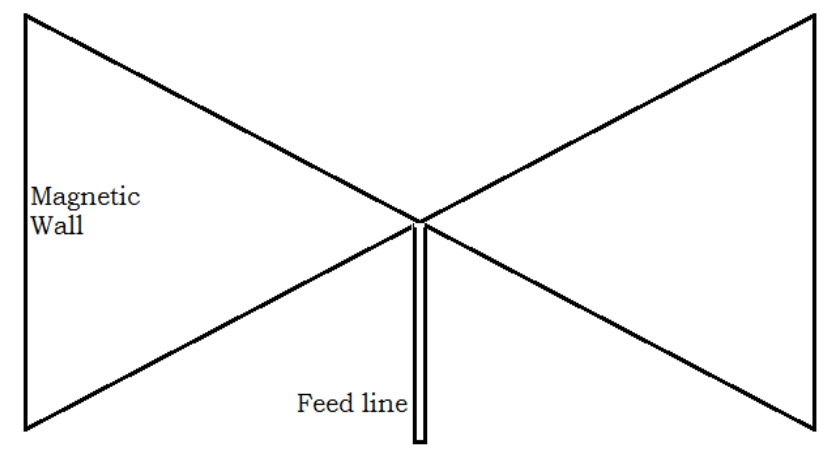

Fig. 1. Biconical antenna.

The resonant frequency corresponding to the many different modes in the MBPA can be calculated by equation (1) [8-9]..

$$
f_{m, n}=\frac{2 c}{3 a \sqrt{\varepsilon_{\mathrm{r}}}} \sqrt{m^{2}+m n+n^{2}}
$$

Where $m, n$ determine the $\mathrm{TM}_{m, n}$ dominant mode; $c$ is the light speed, $\mathrm{m} / \mathrm{s} ; \varepsilon_{\mathrm{r}}$ is the dielectric constant of the substrate; and, $a$ is the length of the antenna arm, $\mathrm{m}$.

It is possible to increase the accuracy of the resonant frequency if the dielectric constant and the length of the arm are replaced by their effective values. The effect of the dielectric constant of the substrate is the major cause of this consideration.

$$
\begin{aligned}
& \varepsilon_{\mathrm{eff}}=\frac{\varepsilon_{\mathrm{r}}+1}{2}+\frac{\varepsilon_{r}-1}{2} \frac{1}{\sqrt{1+\frac{12 d}{a}}} \\
& a_{\mathrm{eff}}=a+\frac{d}{\sqrt{\varepsilon_{r}}}
\end{aligned}
$$

Where $\varepsilon_{\text {eff }}$ is the effective dielectric constant of the substrate; $a_{\text {eff }}$ is the effective length of the arm, m; and, $d$ is the thickness of the substrate, $\mathrm{m}$.

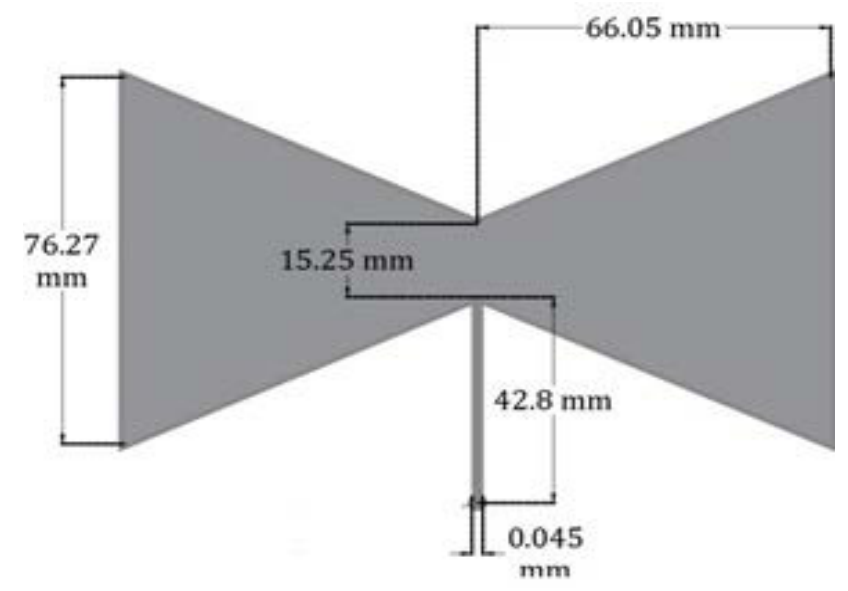

Fig. 2. Layout of the antenna.
The geometry of the MBPA is illustrated in the Figure 2, where the antenna has been matched by a microstrip transformer to a $50 \Omega$ coaxial line and the triangles are folded in the patch.

This design and simulations were carried out for a resonant frequency nearly equal to $2.5 \mathrm{GHz}$ considering the use of a FR-4 substrate. In the results section, the antenna was performed with a different substrate, which dielectric constant is approximately equal to 6.1 . The final resonant frequency was $2.11 \mathrm{GHz}$ approximately, as has been mentioned above.

The dimensions of the proposed antenna are shown in the Table 1, where the resonant frequency is included in both: with the ideal and the effective dielectric constants and the effective length of the antenna arms.

Table 1. Parameters obtained in the antenna design.

\begin{tabular}{|c|c|}
\hline Parameter & Value \\
\hline$a$ & $76.27 \mathrm{~mm}$ \\
\hline$u$ & $66.05 \mathrm{~mm}$ \\
\hline$c$ & $15.25 \mathrm{~mm}$ \\
\hline$f_{\mathrm{r}}$ & $2.5 \mathrm{GHz}$ \\
\hline$\varepsilon_{\text {eff }}$ & 4.22 \\
\hline$a_{\mathrm{eff}}$ & $77 \mathrm{~mm}$ \\
\hline$f_{\mathrm{r}}$ & $2.52 \mathrm{GHz}$ \\
\hline
\end{tabular}

The microstrip transmission line is necessary to match the antenna [1-3], where the thickness of the substrate is approximately $1.544 \mathrm{~mm}$ and the length of this strip line is equal to a quarter of the wavelength, see Figure 3. The coaxial connector is located at the end of the microstrip line on the ground plane.

Fig. 3. Profile of the microstrip antenna.

\section{Results}

In this section, the most important electrical and radiation features of the antenna are presented. In the first stage, the antenna was modeled and simulated using High Frequency Simulation Structure (HFSS). The Figure 4 illustrates the field pattern on the antenna surface in the dominant $\mathrm{TM}_{01}$ mode. 


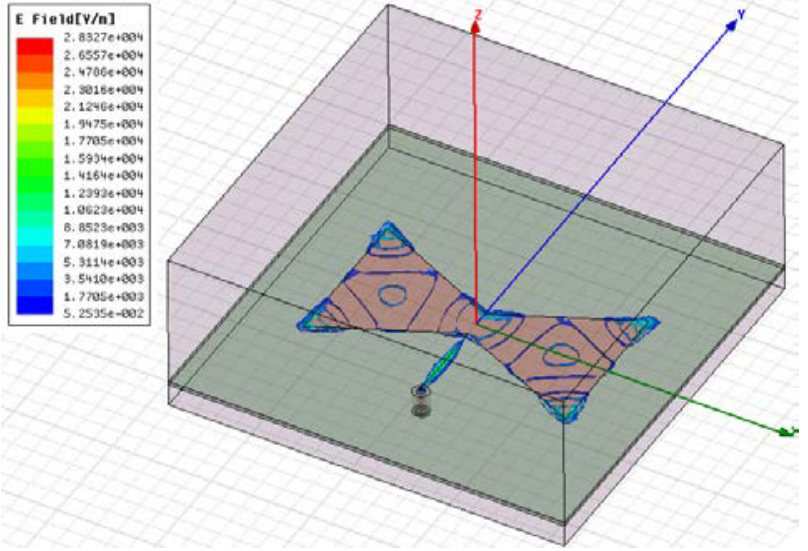

Fig. 4. Simulated model of the antenna.

The simulation process includes the optimization of the structure. In this stage, the antenna patterns and $S_{11}$ parameter are the most important results. The Figure 5 shows the matching interval of the antenna (from 2.05 $\mathrm{GHz}$ to $2.1 \mathrm{GHz}$ ).

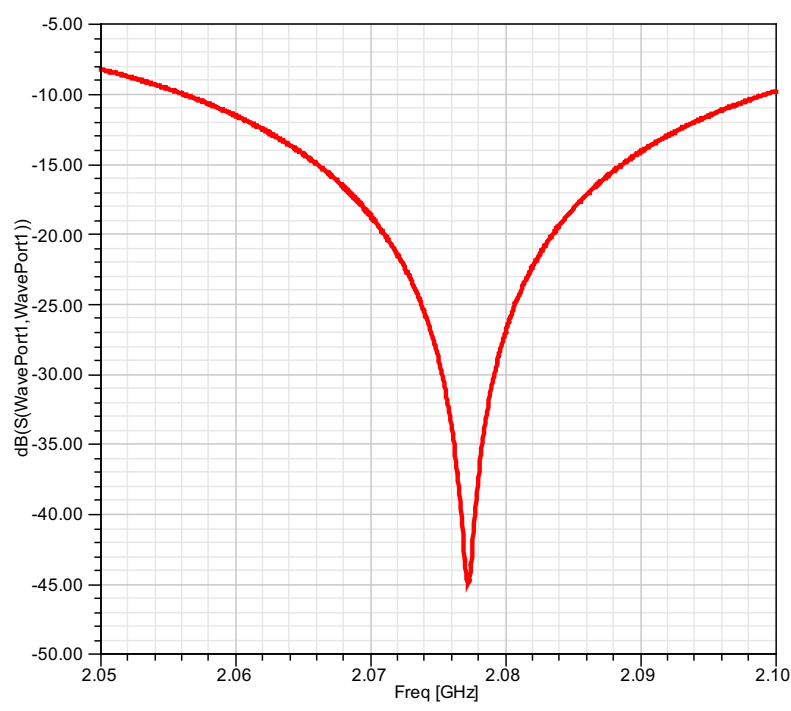

Fig. 5. $\mathrm{S}_{11}$ Parameter in the matching frequency interval of the antenna.
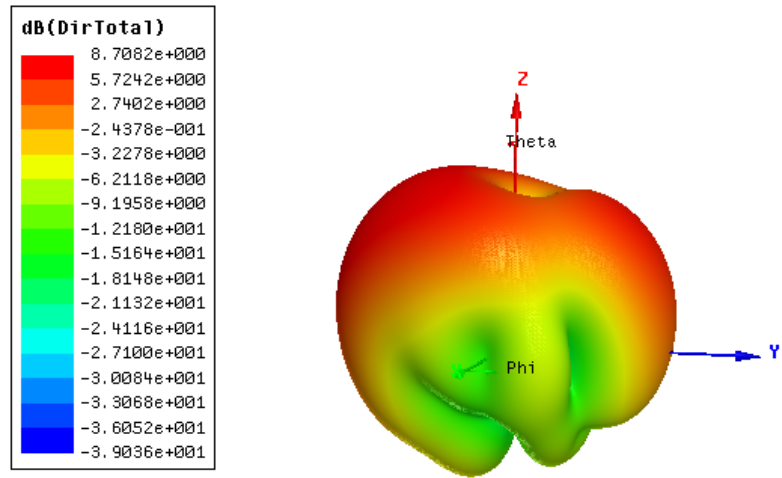

Fig. 6. Simulated 3-D antenna pattern at the far-field zone.
The simulated 3-D antenna pattern at the far field zone is shown in the Figure 6, where the corresponding antenna gain is closed to $8.7 \mathrm{~dB}$.

The 3-D antenna pattern can be divided in two radiation patterns. These patterns could be seen from Figure 7, for $\phi=0^{\circ}$. The simulated radiation pattern in the E-Plane for $\phi=90^{\circ}$ is plotted in the Figure 8. In both antenna patterns, it is clear that the beamwidth is greater than $100^{\circ}$, this characteristic is desirable in almost all the mobile radio-communication systems.

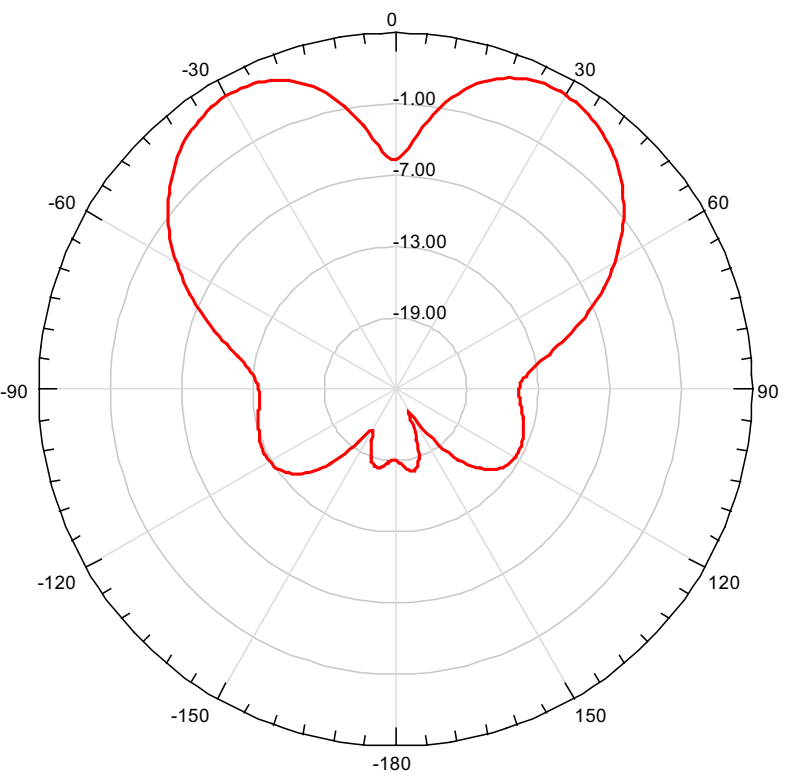

Fig. 7. Simulated radiation pattern in the E-Plane, for $\phi=0^{\circ}$.

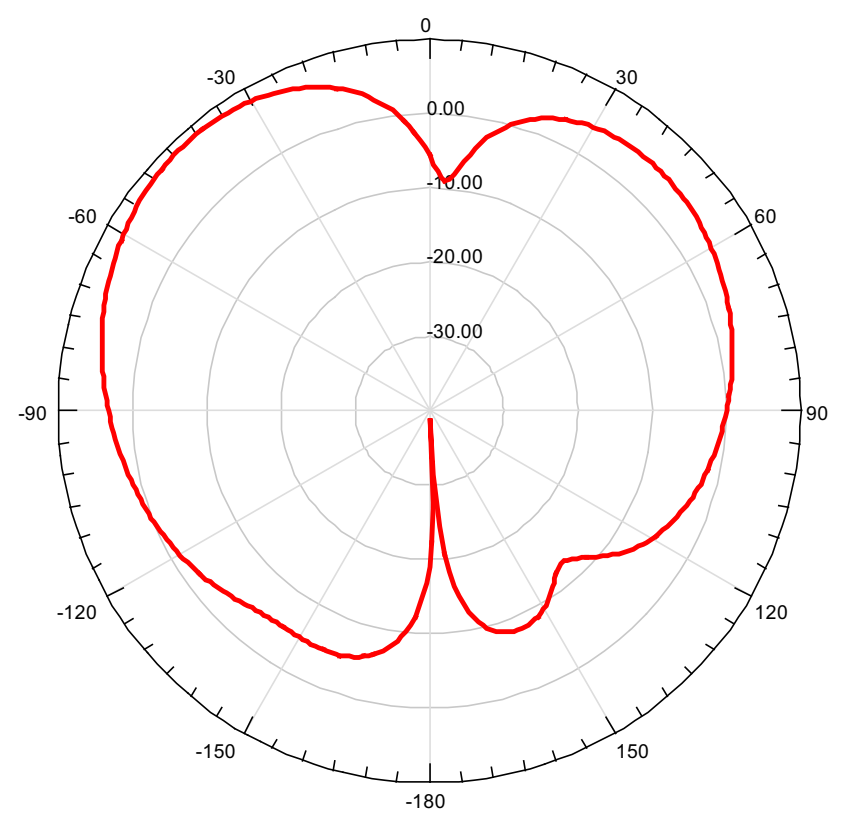

Fig. 8. Simulated antenna pattern in the E-Plane for $\phi=90^{\circ}$.

Based on the simulation results, the MBPA was built using a PCB with a dielectric constant $\varepsilon_{\mathrm{r}} \approx 6$, as it is shown in Figure 9. The computational tool (HFSS) 
employs a finite-element numerical method, which offers a better agreement between simulated and experimental results. Other software choices in simulation of electromagnetic structures like Numerical Electromagnetic Code (NEC) or ENSAMBLE use the method of moments, where the antenna is modeled with surfaces instead of volumetric objects.

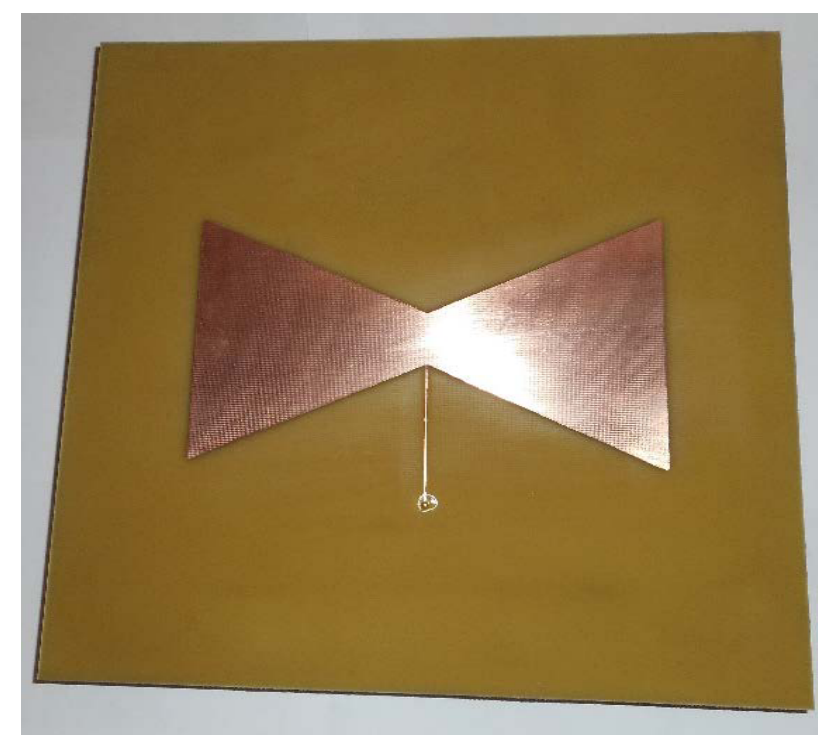

Fig. 9. MBPA printed in a PCB with a dielectric constant nearly equal to 6 .

The return loss of the proposed antenna is shown in Figure 10. The measured frequency interval where the antenna matching is less than $-10 \mathrm{~dB}$ is almost the same in comparison with the corresponding in the simulation results.

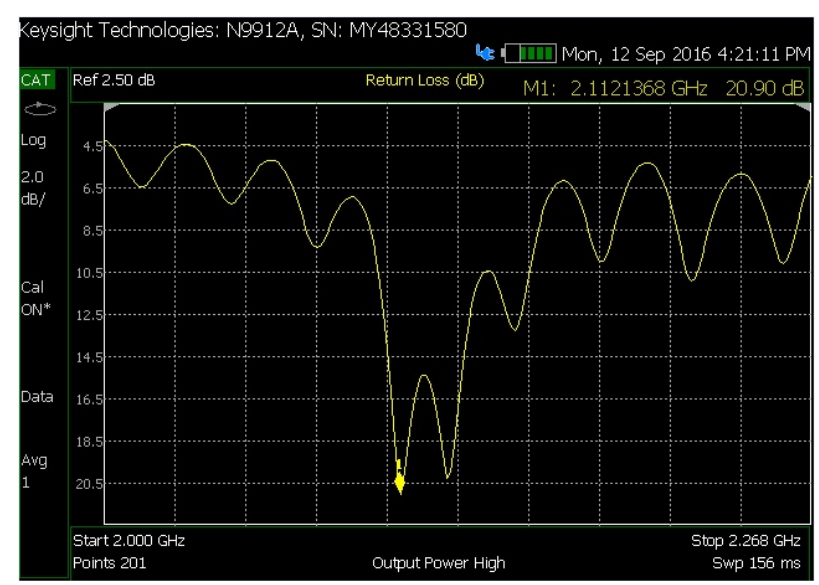

Fig. 10. Measured return loss for MBPA.

The measured far-field 3-D radiation pattern of the MBPA is shown in Figure 11. This result was obtained by interpolation using near-field evaluation equipment. It is obvious that simulation and measured antenna patterns look similar. The size of the antenna shown in the Figure
9 is compatible with the near-field equipment, which is a good choice in comparison with anechoic chambers.

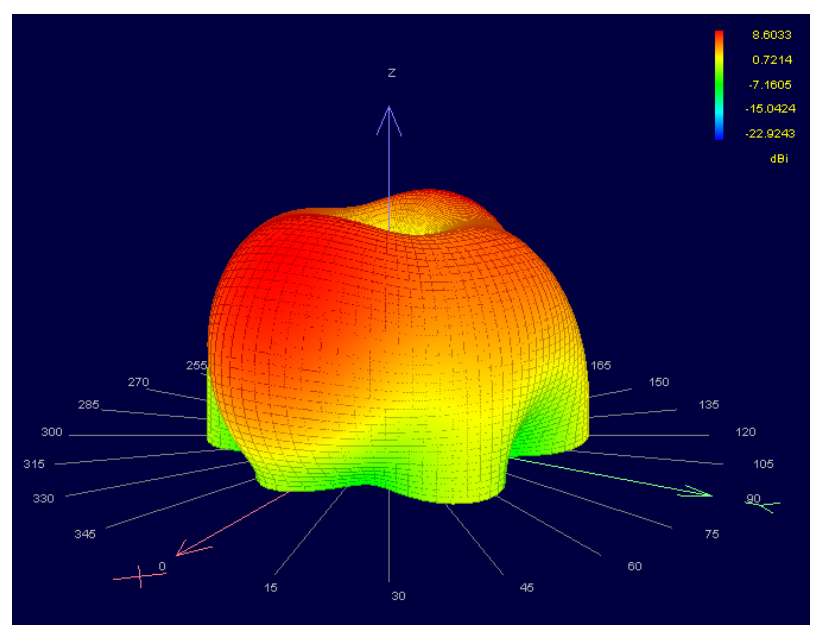

Fig. 11. Measured 3-D antenna pattern for MBPA.

The antenna has also a second resonant frequency at one half of the main resonant frequency $(1.09 \mathrm{GHz}$ instead of $2.11 \mathrm{GHz}$ ). This feature can be used in applications for a lower carrier frequency with the aim of reducing the electrical size of the structure, see Figure 12.

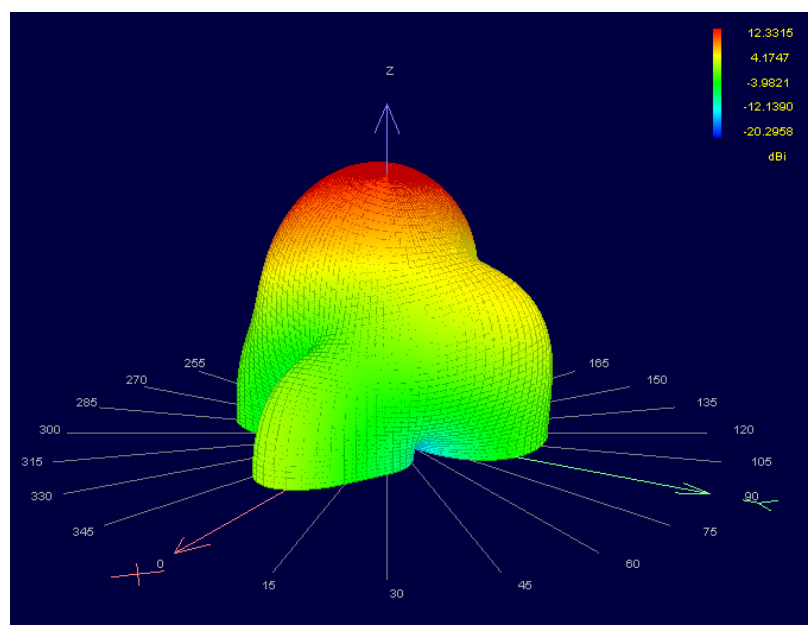

Fig. 12. Measured 3-D antenna pattern for MBPA (1.09 GHz).

\section{Conclusions}

A microstrip bowtie patch antenna was designed and implemented for indoor radio-communications. The proposed antenna has been simulated and measured in order to compare the results, where both numerical results are closed. The antenna gain $(\approx 8.6 \mathrm{dBi})$ is enough to make feasible the link budget in indoor radiocommunications systems. In addition to this, the 
beamwidth is also compatible with this kind of applications.

The measured $\mathrm{S}_{11}$ parameter shows a good agreement with the simulation results and it is clear that the MBPA has a higher bandwidth in comparison with rectangular and circular microstrip patch antennas.

Finally, it is important to emphasize that the MBPA could be used at $1.09 \mathrm{GHz}$, as a consequence of the total length of the structure. In this case, the beamwidth is narrowed down to $60^{\circ}$ approximately.

\section{References}

1. C. A. Balanis, Antenna Theory, 811-876 (2005).

2. D. M. Pozar, Microwave Engineering, 137-146 (1995).

3. J. Volakis, Antenna Engineering Handbook, 7, 1-29 (2007).

4. K. R. Mahmoud, PIER, 100, 105-117, (2010).

5. C. N. Ng, S. Uysal and M. S. Leong, RAWCON'98, 205-207 (1998).

6. B. Harchandra, R. Singh, IJETT, 18, 171-175 (2014).

7. K. W. Loi, S. Uysal, M. S. Leong, IEE Proc. Microw. Antennas Propag., 145, 137-140 (1998).

8. M. Roslee, K. S. Subari, I. S. Shahdan, RFM'2011, 430-433 (2011).

9. C. Guo, R. C. Liu, GPR'2010, 1-5 (2010). 